\title{
First reports of adverse drug reactions
}

(c) Springer Nature Switzerland AG 2019

Table 1 contains an overview of first published case reports of adverse drug reactions identified in the international literature in recent weeks by Reactions Weekly, the Adis drug safety newsletter. Reactions Weekly provides summaries of adverse drug reaction news sourced from journals, scientific meetings, media releases, regulatory agency websites, and bulletins from the National Centers that participate in the WHO International Drug Monitoring Programme.

\section{Table 1 First published reports of adverse drug reactions recently identified by Reactions Weekly}

Drug and adverse reaction

Alectinib: acute hepatitis (serious)

Avelumab: immune thrombocytopenia and anaemia (serious)

Dimethyl fumarate: erythema nodosum

Ibrutinib: anterior chamber fibrinoid syndrome (serious)

Lenvatinib: acquired perforating dermatosis presenting as perforating folliculitis and elastosis perforans serpiginosa

Lithium carbonate: congenital diaphragmatic hernia in a neonate following in utero exposure (serious)

Methylprednisone: spontaneous aorto-oesophageal fistula (serious)

Mianserin: acute generalised exanthematous pustulosis (serious)

Ramucirumab: trachea-oesophageal fistula (serious)

Ruellia bahiensis: fulminant hepatic failure (serious)

Shilajit (mumijo) [herbal supplement]: pseudohyperaldosteronism during pregnancy (serious)

\section{References}

Zhu VW, Lu Y, Ou S-HI. Severe acute hepatitis in a patient receiving alectinib for $A L K$-positive non-small-cell lung cancer: histologic analysis. Clin Lung Cancer. 2019;20(1):e77-80. https://doi.org/10.1016/j.cllc.2018.09.006

Kratzsch D, Simon J-C, Ponitzsch I, et al. Lethal thrombocytopenia in a patient treated with avelumab for metastatic Merkel cell carcinoma [letter]. J Dtsch Dermatol Ges. 2019;17(1):73-5. https://doi.org/10.1111/ddg.13722

Algahtani H, Shirah B, Marghalani S, et al. Erythema nodosum in a patient with multiple sclerosis on dimethyl fumarate. Mult Scler Relat Dis. 2019;28:155-8. https://doi.org/10.1016/j.msard.2018.12.032

Kolomeyer AM, Hwang CK, Kim BJ. Anterior chamber fibrinoid syndrome after cataract extraction in a patient on ibrutinib for B-cell chronic lymphocytic leukemia: a case report and review of the literature. J Med Case Rep. 2018;12(1):349. https://doi.org/10.1186/ s13256-018-1822-9

Sato-Sano M, Cury-Martins J, Macarenco R, et al. Perforating dermatosis associated with multikinase inhibitors: report of two cases, including one associated with lenvatinib. J Dtsch Dermatol Ges 2018;16(12):1486-9. Ihttps://doi.org/10.1111/ddg.13704

Hosseini S-H, Mousavi S-A, Rashidi H. Congenital diaphragmatic hernia following usage of lithium carbonate; is lithium a teratogen? Iranian J Pediatr. 2010;20(1):127-30. https://www.ncbi.nlm.nih.gov/pmc/articles/PMC3446010/

Ju TR, Lee CC, Lin YC. Aortoesophageal fistula causing massive gastrointestinal bleeding and death in a patient with dermatomyositis: a case report. Am J Case Rep. 2018;19:1025-9. https://doi.org/10.12659/AJCR.911441

Allouchery M, Hainaut Wierzbicka E, Frouin E, et al. Acute generalized exanthematous pustulosis induced by mianserin. Eur J Dermatol. 2018;28(4):556-7. http://doi.org/10.1684/ejd.2018.3339

Lee Y-L, Hsu J-F, Yang C-J. Tracheoesophageal fistula in a patient with advanced non-small cell lung cancer who received chemoradiotherapy and ramucirumab [letter]. J Thorac Oncol. 2019;14(1):e17-18. https://doi.org/10.1016/j.jtho.2018.08.2031

Santos Junior GO, Ferreira Filho RP, Foulon LMC, et al. Fulminant hepatic failure caused by herbal supplies. Brazil J Pharmaceut Sci. 2018;54(3). http://dx.doi.org/10.1590/s2175 $-97902018000317418$

Stavropoulos K, Sotiriadis A, Patoulias D, et al. Pseudohyperaldosteronism due to mumijo consumption during pregnancy: a licorice-like syndrome. Gynecol Endocrinol. 2018;34(12):1019-21. https://doi.org/10.1080/09513590.2018.1480713

An event is serious (US FDA MedWatch definition) when the patient outcome is death, life threatening, hospitalization, disability, congenital anomaly or requires intervention to prevent permanent impairment or damage 